\title{
In silico analysis of the dog genome identifies Canine Endogenous Retroviruses (CfERVs)
}

\author{
Álvaro Martínez Barrio'1, Marie Ekjerlund², Göran O Sperber ${ }^{3}$, \\ Jonas Blomberg4, Erik Bongcam-Rudloff1,2 and Göran Andersson*2
}

\begin{abstract}
Address: ${ }^{1}$ The Linnaeus Centre for Bioinformatics, Uppsala University, Biomedical centre, P.O. Box 598, SE-75124 Uppsala, Sweden, ${ }^{2}$ Department of Animal Breeding and Genetics, Swedish University of Agricultural Sciences, Biomedical centre, P.O. Box 597, SE-751 24 Uppsala, Sweden, ${ }^{3}$ Department of Neuroscience, Physiology, Uppsala University, Uppsala, Sweden and ${ }^{4}$ Section of Virology, Department of Medical Sciences, Uppsala University, Uppsala, Sweden

* Corresponding author
\end{abstract}

from Frontiers of Retrovirology: Complex retroviruses, retroelements and their hosts

Montpellier, France. 21-23 September 2009

Published: 24 September 2009

Retrovirology 2009, 6(Suppl 2):P7 doi:10.1 186/1742-4690-6-S2-P7

This abstract is available from: http://www.retrovirology.com/content/6/S2/P7

(c) 2009 Barrio et al; licensee BioMed Central Ltd.

A whole dog genome analysis was perfomed using RetroTector $^{\circ}$ to identify and define the complexity of canine endogenous retroviruses (CfERV). Results show that all dog chromosomes contain CfERV integrations. Furthermore, the integration pattern was shown to be uneven with some regions essentially devoid of integrations and other regions having large amounts of CfERV integrations. The dog may well have been effective in protecting its genome from integrations of most types of endogenous retroviruses. Compared to mouse, chimpanzee and human, dog has substantially lower amounts of ERVs. We identified a total of 416 ERVs, which is only approximately a fifth of the amount of HERVs found in the human genome. Phylogenetic analysis showed that the vast majority of CfERVs cluster with the gammaretrovirus genus $(\mathrm{n}=318)$. The second most common group was the beta-retroviruses $(n=27)$. This pattern is similar in other vertebrates. In addition, three spuma-like and four gypsylike CfERVs were identified. The latter group is rare in vertebrate genomes. Moreover, we identified a group of 55 CfERVs with mixed phylogenetic relationship to any known retroviral genera. The integration pattern of CfERVs was analyzed in relation to genes in their vicinity and a substantial fraction of CfERV was found within annotated genes and within $100 \mathrm{~kb}$ from annotated dog genes. Interestingly, a group of recently integrated CfERVs with similarity to HERV-Fc1 was found. Several of them had all or nearly all major reading frames open, a sign of functionality. Therefore, some of these CfERVs may have potential for active retrotransposition and thus actively contribute to the plasticity of canine genomes. The differential distribution and amounts of ERVs in the dog genome compared to genomes in the primate and rodent clades suggest species-specific mechanisms of purging different classes of ERVs. 